\title{
Sorting on Single-Channel Wireless Sensor Networks *
}

\author{
Jacir L. Bordim, Koji Nakano, and Hong Shen \\ School of Information Science \\ Japan Advanced Institute of Science and T ec hnology \\ 1-1, Asahidai, Tatsunokuchi, Ishika wa 923-1292, Japan
}

\begin{abstract}
A wireless sensor network is a distribute dsystem which consists of a base station and a number of wireless sensors nodes endowed with radio transceivers. The main contribution of this work is to present a sorting protocol for multi-hop wireless sensor networks. Our protocol sorts $n$ elements which are initially lo ade $\mathrm{d} n \mathrm{n}$ sensor nodes that are organize din a two-dimensional plane of size $\sqrt{n} \times \sqrt{n}$. The sorting protocol proposed here sorts the $n$ elements in $O(r \sqrt{n})$ time slots when $\sqrt{n}>r$, where $r$ is the transmission range of the sensor nodes.
\end{abstract}

keywords: wir eless sensor networks, sorting, bitonic sorting, sensing devices

\section{Introduction}

A Wireless Sensor Network (WSN, for short) is a distributed system consisting of a base station and a $\mathrm{n}$ umber of tiny wireless sensing devices that integrate microsensing and short-range communication capabilities. When deployed in large $n$ umbers, these devices can measure aspects of the ph ysical environment in great detail. The data being sensed by the sensor nodes in the netw ork is en tually transferred to a base station, where the information can be accessed.

In a single-hop WSN, a sensor node can directly communicate with any other sensor node, whereas in a multi-hop WSN, the communication between tw o sensor nodes may involve a sequence of hops through a chain of pairwise adjacent sensor nodes. There is a single-hop communication betw eenthe base station and the sensor nodes, while the communication among the sensor nodes can be either single or multi-hop.

*Work partially supported by The Hori Information Science Promotion Foundation.
There are several possible models for WSN's, in this w ork consider WSN's where all the sensor nodes in the netw ork are fixed, short-ranged and homogeneous. We assume that the base station and all the sensor nodes have a local clodk that keeps synchronous time, perhaps by interfacing with the base station or with a GPS system [13]. All sensor nodes run the same protocol and can perform computations on the data being sensed. As customary, time is assumed to be slotted and all transmissions take place at slotted boundaries $[6,8]$. We employ the commonly-accepted assumption that when tw o or more sensor nodes whid are in the transmission range of each other transmit in the same time slot, the corresponding pac ketscollide and are garbled beyond recognition.

In this work we address the sorting problem, where $n$ elements are stored in $n$ sensor nodes which are arranged in a tw o-dimensional square plane of size $\sqrt{n} \times \sqrt{n}$. Sorting is a fundamental problem with an extensive theory and a wide range of practical applications. It is kno wn that a sequetial algorithm takes at least $\Omega(n \log n)$ time to sort a sequence of $n$ elements and that optimal algorithms exist which achieve $O(n \log n)$ time [3]. Also, many optimal parallel sorting algorithms have been reported in the literature for differen t parallel ardhitectures, such as the Parallel Random Access Machine (PRAM) and the Reconfigurable Mesh (RM) [4, 14].

The sorting protocol proposiad this work follows from the work of Nassimi and Sahni [15], which is an adaptation of the bitonic sort. It was sho wn in $[1$ that $n^{2}$ elements can be sorted in $O(n)$ time on a MeshConnected $\mathrm{P}$ arallel Computer. Our sorting protocol sorts $n$ elements which are initially stored in $n$ sensor nodes in $O(r \sqrt{n})$ time slots, for $r<\sqrt{n}$, where $r$ is the transmission range of the sensor nodes. F or short-transmission ranges (i.e., small values of $r$ ), our sorting algorithm matches the time complexity of the algorithm proposed by Nassimi and Sahni [15], which 
is optimal. How ever, our protocol can tak as much as $O\left(r^{2}\right)$ time slots if $r \approx \sqrt{n}$. This is due to the fact that with a large transmission range, the number of sensor nodes that can transmit concurrently decreases, since they ha veto lay well apart from each other to a void in terference. As a consequence, the performance of our protocol decreases. Nevertheless, the assumption that the sensor nodes employ short-range wireless communications, less than 100 meters, is customary [5, 12] since it allo ws the use of tiny and low-powered radio transceivers. In addition, short-range transmissions allows greater radio channel reuse, th us increasing the aggregate bandwidth available. The Piconet [7] project is dev eloping a prototype embedded netw ork that uses short-range (5 meters) radio communications. The sensor nodes in the WSN can use Piconet to enable wireless connectivity. Therefore, one can argue that WSN's will employ short-range radio transmissions to allow communication among the sensor nodes.

\section{Model and Problem Definition}

The base station is assumed to be equipped with a large antenna which covers a wide area, so that it can monitor all the sensor nodes under its coverage area. The computation among the sensors is performed in coordination with the base station. A sensor node in a single-hop WSN can tune to a channel to send/receive a packet. A t the end of a timeslot, the status of the channel can be: $(i)$ NULL, no packet has been driven into the channel in the current time slot; (ii) SINGLE,exactly one packet has been driven into the channel in the current time slot; or (iii)COLLISION, two or more packets ha ve been drien in to the $\mathrm{c}$ hannel in the current time slot.

When a sensor node transmits a packet with pow er $r$, the signal will be strong enough for other sensors to hear it within the Euclidean distance $r$ from the sensor node that originates the packet. Let us observe the channel status of a sensor node. F or this purpose, let $A$ be a sensor node in a WSN and let $S$ be the unique sensor node broadcasting in a giv en time slot. The channel status of $A$ is NULL only if $A$ is outside the transmission range of $S$. Otherwise, if $A$ is within the radio transmission of $S$, its channel status is SINGLE. Now, let us consider the case in which two or more sensor nodes are broadcasting at the same time. Clearly, if their transmissions do not interfere (i.e., do not overlap), the channel status of $A$ is as discussed above. In case of overlapping transmissions, the channel status is as follows. The channel status of sensor node $A$ is COLLISION if it is within radio transmission of tw $o$ or more sensors. Therefore, a sensor node is ensured to receive a pac ket,only if it lies in the transmission range of the source node and there is no interference from other broadcasts.

In this w ork, w eassume that the sensor nodes in the WSN are organized as a tw odimensional square plane of size $\sqrt{n} \times \sqrt{n}$ with coordinates $(x, y),(1 \leq$ $x, y \leq \sqrt{n})$. The plane can be viewed as $n$ cells of unit size $1 \times 1$. Let $C(x, y),(1 \leq x, y \leq \sqrt{n})$, denote a cell consisting of all points $\left(x^{\prime}, y^{\prime}\right),\left(x \leq x^{\prime}<x+1 ; y \leq\right.$ $\left.y^{\prime}<y+1\right)$. Suppose that each cell $C(x, y)$ has a sensor denoted by $S_{x, y}$. Throughout this work we assume that each sensor node $S_{i, j},(1 \leq i, j \leq \sqrt{n})$, kno ws its cell location within the grid. Clearly, for any tw o sensors located in adjacent cells, the farthest distance between them is $\sqrt{5}$. Hence, to ensure the communication betw een adjacent sensors, a pac ketmust be transmitted with pow er of at least $\sqrt{5}$ to cover a region of $\sqrt{5} \approx 2.24$. Similarly, sensors in diagonally adjacent cells ha ve distanceof at most $2 \sqrt{2}$. Thus, a sensor has to transmit with enough pow er to coer an area of at least $2 \sqrt{2} \approx 2.83$ to ensure communication with its neighbors. If the sensors on a WSN of size $\sqrt{n} \times \sqrt{n}$ can broadcast with sufficient power to cover an area of $\sqrt{2 n}$, then, any pair of sensors can directly communicate, that is, the WSN essen tially allows a single-hop communication. In other words, if the sensors with transmission range $r$ are allocated on a WSN of size $\frac{\sqrt{2} r}{2} \times \frac{\sqrt{2} r}{2}(\approx 0.71 r \times 0.71 r)$, then a single-hop communication is ensured.

Assume that $n$ elements are stored in $n$ sensor nodes, where each sensor holds exactly one element. Also, let $S_{i}$ be the sensor node with index $i,(1 \leq i \leq n)$. The sorting problem is defined to be the problem of moving the $i$ th smallest element to the sensor $S_{i}$, for all $i=1,2, \ldots, n$. Our sorting protocol is based upon the w ork of Nassimi and Sahni [15], which in turn is an adaptation of the Batcher's bitonic sort algorithm [9]. The bitonic sort algorithm sorts a bitonic sequence in to nondecreasing order. A sequence $\left\{a_{1}, a_{2}, \ldots, a_{2 n}\right\}$ is said to be bitonic if either $(i)$ there is an integer $j$ such that $a_{1} \leq a_{2} \leq \ldots \leq a_{j} \geq \ldots \geq a_{2 n}$, or $(i i)$ the sequence does not satisfy condition $(i)$ but can be shifted cyclically until condition $(i)$ is satisfied $[4,9]$.

An interesting propriety of the sorting algorithm proposed in [15], is that operations like comparing and exc hanging need only to be performed among the elements that belong to the same row or column. Thus, before presenting the details of our protocols, let us first consider an array of size $1 \times n$ consisting of $n$ adjacent cells. Suppose that the transmission range $r$ of each sensor node equals to $n$. Obviously, the maximum distance betw een the sensor nodes that are located at the extreme positions of the array (i.e., $S_{1}$ and $S_{n}$ ) is 
$\sqrt{n^{2}+1}$. In suc $\mathrm{h}$ a case, a single-hop communication cannot be ensured between $S_{1}$ and $S_{n}$, since the maximum distance betw een the oxceedsr. On the other hand, a single-hop communication can be ensured betw een $S_{1}$ and $S_{n-1}$ (and also with an yother sensor node that lies between them), since the farthest distance betw een themis less than $r$. It should be clear from the above that if collision is to be av oided at $S_{n-1}$, any other sensor node that broadcasts along with $S_{1}$ must be apart from $S_{n-1}$ of a distance greater than $r$.

\section{Sorting on Single-hop WSN's}

In this section w e present a sorting protocol for single-hop WSN's. Suppose that a WSN has $m$ sensor nodes, where all of them lie in the transmission range of eac $\mathrm{h}$ other, and ead sensor has a unique ID in the range $[1, m]$. Let $S_{i}$ denote the sensor node with ID $i$ $(1 \leq i \leq m)$, that holds an element $x_{i}$. The $m$ elements can be sorted in $2 m$ time slots as follows. F or eac $\mathrm{h}$ time slot $i,(1 \leq i \leq m)$, the sensor node $S_{i}$ broadcasts $x_{i}$ on the channel and each sensor node $S_{j},(1 \leq j \leq m)$, monitors the channel to receiv e $x_{i}$. By comparing $x_{j}$ to $x_{i}$, each sensor node $S_{j}$ can compute the rank of its element. Once the ranking of each element has been computed, the elements are routed to their final destination, which incurs in additional $m$ time slots. The follo wing lemma summarizes the above discussion:

Lemma 1 The elements on a single-hop WSN consisting of $m$ sensor no des, where each sensor no deholds one element, can b e sortd in $2 m$ time slots.

Clearly, the above result is optimal considering that at any given time slot, only one sensor node can transmit on the channel. Otherwise, a collision occurs and the pac ketsare lost. If the $m$ sensor nodes are arranged in a $\sqrt{m} \times \sqrt{m}$ array, w ecan rank and sort the elements in a column/row into either increasing or decreasing column/row order in $2 \sqrt{m}$ time slots.

Corollary 1 When $m$ sensor nodes are arr angel in a $\sqrt{m} \times \sqrt{m}$ single-hop WSN, a single column/row can be sorted in $2 \sqrt{m}$ time slots.

\section{Sorting on Multi-hop WSN's}

This section presents a sorting protocol for multihop WSN's. T o begin with, the $\sqrt{n} \times \sqrt{n}$ array is partitioned into $\frac{\sqrt{n}}{2 r} \times \frac{\sqrt{n}}{2 r}$ groups of size $2 r \times 2 r$, which are further divided into 16 blocks of size $\frac{r}{2} \times \frac{r}{2}$. Note that there is a single-hop communication among the sensor nodes located within each block. F urthermore, a sensor node within a block can communicate with a sensor node in a neighboring adjacent block that occupies the same relative position within that block. We assume that $n$ and $r$ are power of two and that $\sqrt{n}>r$. The elements in a row or in a column can be sorted either in increasing or decreasing order. We say that an element is rejectedif it is against the order in which the array is being sorted. The order in which the array is to be sorted is defined at a later stage in the main protocol. Our sorting protocol comprehends a number of sub-protocols whose details are discussed below.

\subsection{Row and Column Sorting}

We begin with a protocol that sorts a bitonic sequence of size $\kappa$, where the $\kappa$ elements are stored in $\kappa$ adjacent sensor nodes. The details of the protocol are spelled out as follows:

Protocol Row-Merge $(\kappa)$

1. Let $S_{i},(1 \leq i \leq \kappa)$, be the sensor node that stores the element $x_{i}$. Also, let $P_{1}=\left\{S_{1}, \cdots, S_{\kappa / 2}\right\}$, and $P_{2}=\left\{S_{\kappa / 2+1}, \cdots, S_{\kappa}\right\}$;

2. if $\kappa=2 r$ then return;

3. Shift the elements from $P_{2}$ to $P_{1}$;

4. P erform a comparison-interc hange on $P_{1}$;

5. Shift the rejected elements from $P_{1}$ to $P_{2}$;

6. In parallel, inv ok eRow-Merge $(\kappa / 2)$ for $P_{1}$ and $P_{2}$;

In eac $h$ iteration of the above protocol, a sequence of size $\kappa / 2$ has to travel $\kappa$ positions, $\kappa / 2$ positions to the left and $\kappa / 2$ positions to the right. Note that an element is ensured to be correctly receiv edby a sensor node that is located $r / 2$ positions from the sender, since the maximum distance betw een them does not exceed $r$. In order to av oid collision with other sensor nodes that are broadcasting at the same time, for each sequence of size $2 r$, only one element is allow ed to transmit in each time slot. Thus, $(\kappa / 2) / 2 r=\kappa / 4 r$ elements, can trav el simultaneously without interfering with each others' broadcast. Hence, a sequence of size $2 r$ tak es $(2 r \kappa) /(r / 2)=4 \kappa$ time slots to trav el $\kappa$ positions. Since we can move the elements of each sequence in parallel, it takes $4 \kappa$ time slots to mov e $\kappa / 2$ elements $\kappa$ positions.

We now turn to number of iterations taken b y Protocol Row-Merge $(\kappa)$ to complete its execution. The protocol returns when $\kappa=2 r$, that is, the protocol will be executed for $\log \kappa-(\log r+2)$ iterations. Thus, the total $\mathrm{n}$ unber of time slots can be computed by:

$$
\sum_{i=\log r+2}^{\log \kappa} \frac{4 \kappa}{2^{(\log \kappa)-i}}=4 \kappa \cdot\left[2-\frac{1}{2^{\log \kappa-\log r-2}}\right]
$$




$$
\begin{aligned}
& =4 \kappa \cdot 2-\frac{4 r}{\kappa} \\
& =8 \kappa-16 r
\end{aligned}
$$

A $t$ this poitt, we have $\frac{\kappa}{2 r}$ groups of sequences of size $2 r$ that still need to be sorted. F or this purpose, Protocol Row-Merge $(\kappa)$ is sligh tly modified sudh that it can sort a row of elements of size $2 r$. The main difference is that within each sequence of size $2 r$ only one sensor is allow edto broadcast at a time. Consequently, w e cannot perform Step 6 in parallel. On the other hand, Step 6 can be executed in parallel for neighboring sequences. Thus, when the size of the input sequence is reduced to $2 r$, we can sort the sequence for each group sequentially until the size of the sequence is reduced to $r / 2$, we then apply Corollary 1 to sort the remaining sequence. The following tw o steps need to be modified in Protocol Row-Merge $(\kappa)$ to sort a bitonic sequence of size $2 r$.

2. if $\kappa=r / 2$ then return;

6. Invoke Row-Merge $(\kappa / 2)$ for $P_{1}$ and $P_{2}$ sequentially;

Note that tw o iterations are sufficient to reduce the size of the sequence to $r / 2$. In the first iteration, a sequence of size $r$ is shifted 4 hops (tw oto the left, compare-interchange and shift back). In the second iteration, a sequence of size $r / 2$ is shifted 2 hops, which takes $2 r$ time slots since we have 2 of such sequences. Then, since asingle-hop comm unication is ensured, a sequence of size $r / 2$ can be sorted in $r$ time slots according to Corollary 1. Thus, it tak es $6+4 r=10 r$ time slots to sort a bitonic sequence of size $2 r$. Altogether, it takes $8 \kappa-6 r<8 \kappa$ time slots to sort a bitonic sequence of size $\kappa$. The follo wing lemma summarizes the abov e discussion:

Lemma 2 The $\kappa$ elements stored in $\kappa$ adjac ent sensor no des on a row on the $\sqrt{n} \times \sqrt{n}$ array can be sorte $d$ into either increasing or decreasing $r$ ow-major oder in less than $8 \kappa$ time slots.

The Protocol Column-Merge $(\tau)$ is defined in a similar way, except that it sorts a bitonic sequence of size $\tau$ that is stored in $\tau$ adjacent sensors in a column of the $\sqrt{n} \times \sqrt{n}$ array.

Lemma 3 The $\tau$ elements stored in $\kappa$ adjac ent sensor nodes on a column of the $\sqrt{n} \times \sqrt{n}$ array can be sorte $d$ into either incr easingor decreasing column-major order in less than $8 \tau$ time slots.

\subsection{Vertical Merge Sort}

The Protocol V ertical-Merge $(\tau, \kappa)$ sorts in to either increasing or decreasing row-major order an arra y of size $\tau \times \kappa$ which is composed of two vertically aligned arrays of size $\tau / 2 \times \kappa$, where one is in increasing rowmajor order and the other is in decreasing row-major order. It has been sho wn in [15] that tw o vertically aligned arrays can be sorted by column-merge follow ed by row-merge sinadl columns are bitonic, and after executing column-merge, all rows are bitonic. For further details, we refer the reader to [15].

Protocol Vertical-Merge $(\tau, \kappa)$

1. for all columns in parallel do Column-Merge $(\tau)$;

2. for all rows in parallel do Row-Merge $(\kappa)$;

The total number of time slots of the Protocol V ertical-Merge $(t, \kappa)$, accordingly to Lemma 3 and Lemma 4, is Vertical-Merge $(\tau, \kappa)=8(\tau+\kappa)$ time slots. Since we can only process one of the $2 r$ lines/columns at a time, the total number of time slots is less than $16 r(\kappa+\tau)$. The above results are summarized in the follo wing lemma.

Lemma 4 Two vertically aligned arrays of size $\tau / 2 \times$ $\kappa$, where one is in increasing $r$ ow-major oder and the other is in decreasing row-major order can be sorted in less than $16 r(\kappa+\tau)$ time slots.

\subsection{Horizontal Merge Sort}

The Protocol Horizontal-Merge $(\tau, \kappa)$ sorts an array of size $\tau \times \kappa$ which consists of tw o horizontally aligned adjacent arrays of size $\tau \times \kappa / 2$. One of these arrays is sorted in to increasing row-major order and the other in to decreasing row-major order. Before showing the details of the Protocol Horizontal-Merge, we first introduce the Protocol TC-Merge $(\tau)$ (Two-Column-Merge), which sorts a bitonic sequence of $2 \tau$ elements stored in a column of $\tau$ adjacent sensors. The bitonic sequence $\left(x_{1}, \cdots, x_{2 \tau}\right)$ is stored in sensor $S_{i}(1 \leq i \leq \tau)$ such that each sensor holds tw oelements, $x_{i}$ and $x_{i+\tau}$, of the bitonic sequence. After sorting, each sensor $S_{i}$ will contain the elements $x_{2 i-1}$ and $x_{2 i}$. The details of the protocol are listed below:

Protocol TC-Merge $(\tau)$

1. Let $S_{i},(i \leq 1 \leq \tau)$, be the sensor node that store the elements $x_{i}$ and $x_{i+\tau}$. Also, let $P_{1}=$ $\left\{S_{1}, \cdots, S_{\tau / 2}\right\}$, and $P_{2}=\left\{S_{\tau / 2+1}, \cdots, S_{\tau}\right\}$

2. Compare-interchange the elements in each sensor;

3. if $\tau=2 r$ then return; 
4. Exchange the rejected elements of $P_{1}$ with the accepted elements of $P_{2}$;

5. In parallel, invok eTC-Merge $(\tau / 2)$ for $P_{1}$ and $P_{2}$;

The proof of correctness of the abo protocol can be found in [15]. The analysis of Protocol TC-Merge $(\tau)$ is similar to the Protocol Row-Merge, except that here the elements are exc hanged instead of being shifted to the left and then to the right. Thus the total number of time slots for the Protocol TC-Merge $(\tau)$ is $8 \tau-16 r$. Proceeding as we did before, another $6 r$ time slots are necessary to reduce the size of the sequence from $2 r$ to $r / 2$. Recall that eac h sensor node now holds tw o elements. Hence sorting each column of size $r / 2$ takes $2 r$ time slots according to Corollary 1 . Thus, Protocol TC-Merge $(\tau)$ takes, altogether, $8 \tau-2 r<8 \tau$ time slots.

Lemma 5 The $\tau$ elements stored in $\tau$ adjac ent sensor nodes on a column of the $\sqrt{n} \times \sqrt{n}$ array can be sorte $d$ into either incr easing or decreasing order in less than $8 \tau$ time slots.

We no w ha e all the necessary tools to present the protocol Horizontal Merge. The details of the protocol are as follows:

Protocol Horizontal-Merge $(\tau, \kappa)$

1. Let $C_{1}, C_{2}, \cdots, C_{\kappa}$ represent the $\kappa$ columns and also let $P_{1}=\left\{C_{1}, \cdots, C_{\kappa / 2}\right\}$, and $P_{2}=$ $\left\{C_{\kappa / 2+1}, \cdots, C_{\kappa}\right\}$

2. Move the elements in $P_{2}$ to the corresponding sensors in $P_{1}$;

3. For each column $C_{1}, \cdots, C_{k / 2}$ perform TC-Merge $(\tau)$

4. Move the rejected elements in $P_{1}$ to the corresponding sensors in $P_{2}$;

5. In parallel, invoke Row-Merge $(\kappa / 2)$ for each of the $2 \tau$ rows, each con taining $\kappa / 2$ adjacent sensors. The $2 \tau$ rows are obtained by splitting each original $\tau$ into two.

Recall that routing the elements $\kappa$ positions, $\kappa / 2$ to the left in step 2 and $\kappa / 2$ to the right in step 4 , take $4 \kappa$ time slots. The total number of time slots of Protocol Horizontal-Merge $(\tau, \kappa)$ is giv en $\mathrm{b} \mathrm{y}$ :

$$
\begin{aligned}
H M(\tau, \kappa) & =T C(\tau)+R M(\kappa / 2)+4 \kappa \\
& =8(\tau+\kappa),
\end{aligned}
$$

where $H M, T C$, and $R M$, stand for HorizontalMerge, TC-Merge and Row-Merge, respectively. The follo wing lemma summarizes the abov e results:
Lemma 6 Two horizontally aligned arrays of size $\tau \times$ $\kappa / 2$, where one is in increasing row-major order and the other is in decreasing row-major order, can be sorted in less than $16 r(\kappa+\tau)$ time slots.

\subsection{WSN-Sort}

We are now in a position to show the sorting protocol that sorts $n$ elements stored in $n$ sensor nodes which are arranged in a tw o-dimensional arry of size $\sqrt{n} \times \sqrt{n}$.

Protocol WSN-Sort $(\sqrt{n}, \sqrt{n})$

1. $\kappa \leftarrow 2 r$;

2. Sort all groups of size $2 r \times 2 r$;

3. while $\kappa<\sqrt{n}$ do

4. Execute Horizontal-Merge $(\kappa, 2 \kappa)$ in parallel for each array of size $k \times 2 k$;

5. Execute Vertical-Merge $(2 \kappa, 2 \kappa)$ in parallel for each array of size $2 \kappa \times 2 \kappa$;

6. $\quad \kappa \leftarrow 2 \cdot \kappa$;

\section{7. end while}

For the protocols Horizontal-Merge and VerticalMerge to work properly, it is necessary to satisfy their initial conditions, that is, some subarrays must be sorted into increasing order and others into decreasing order. The order into which the array has to be sorted in steps $\mathbf{2}$ and $\mathbf{5}$ is defined by $\left\lfloor\frac{j-1}{\kappa}\right\rfloor$, and by $\left\lfloor\frac{i-1}{\kappa}\right\rfloor$ for step 4 , where $i$ and $j,(1 \leq i, j, \leq \sqrt{n})$, represent the sensor's row and column indexes, respectively. If the result is even for all sensors on which comparisonin terchanges are being executed, then the subarray is sorted into increasing row-major order, otherwise, it is sorted into decreasing row-major order. We now turn to analysis of the number of time slots taken $\mathrm{b} y$ protocol Sorting. Clearly, step $\mathbf{2}$ can be computed in $O\left(r^{2}\right)$ time slots. The number of time slots for while-loop is is given $\mathrm{b} y$ :

$$
\begin{aligned}
S(\sqrt{n}, \sqrt{n})=S\left(\frac{\sqrt{n}}{2}, \frac{\sqrt{n}}{2}\right) & +H M\left(\frac{\sqrt{n}}{2}, \sqrt{n}\right) \\
& +V M(\sqrt{n}, \sqrt{n}) \\
& =S\left(\frac{\sqrt{n}}{2}, \frac{\sqrt{n}}{2}\right)+56 r \sqrt{n} \\
\leq & 112 r \sqrt{n} \\
& =O(r \sqrt{n}),
\end{aligned}
$$

where $S, V M$, and $H M$, stand for WSN-Sort, $\mathrm{V}$ ertical-Merge, and Horizontal-Merge, respectively . 
Thus, for $r<\sqrt{n}$, the total number of time slots to sort an array of $\sqrt{n} \times \sqrt{n}$ elements, where eac h element is stored in a sensor node, is $O(r \sqrt{n})$ time slots.

Lemma 7 L et a WSN consist of $n$ elements stored in $n$ sensor no des, which are arrange din a twodimensional array of size $\sqrt{n} \times \sqrt{n}$. When $\sqrt{n}>r$, the $n$ elements can b e sortd in $O(r \sqrt{n})$ time slots.

When $r \geq \sqrt{n}$, the tw o-dimensionalarra y will be already sorted after step $\mathbf{2}$, and hence, it takes $O\left(r^{2}\right)$ time slots to sort the arra y. The follo wing corollary summarizes this discussion.

Corollary 2 Let a WSN consist of $n$ elements stored in $n$ sensor no des, which are arrange din a twodimensional array of size $\sqrt{n} \times \sqrt{n}$. When $r \geq \sqrt{n}$, the $n$ elements can $b$ e sortd in $O\left(r^{2}\right)$ time slots.

\section{Conclusions}

In this work we presented a sorting protocol for wireless sensor netw orks. The sorting protocol discussed in here is an adaptation of the parallel sorting algorithm proposed by Nassimi and Sahni [15], which is based on Batcher's bitonic sort algorithm [9]. Our protocol sorts $n$ elements which are initially loaded in $n$ sensor nodes arranged in a tw o-dimensional plane of size $\sqrt{n} \times \sqrt{n}$ in $O(r \sqrt{n})$ time slots without the need of inv olving the base station. We ha vealso shown that future applications of wireless sensor net w orksare very likely to employ short-range radio communications (i.e., small $r)$. If this is the case, our protocol matches the time complexity of the optimal sorting algorithm proposed in [15]. We ha ve also show an optimal sortiraggorithm for single-hop WSN's. How ev er,it remains to be shown whether or not our results are optimal when $1 \ll r \ll \sqrt{n}$.

\section{References}

[1] Abramson, N., Multiple A ccessCommunications: Foundations for Emerging Technolgies, IEEE Press, New York, 1993.

[2] Abramson, N., Multiple ac cess in wir eless digital networks, Proceedings of the IEEE, 82, (1994), 1360-1370.

[3] Aho, A. V., Hopcroft, J. E., and Ullman, J. D., The Design and Analysis of Computer Algorithms, Addison-Wesley, 1974.

[4] Akl, S. G., Parallel Sorting Algorithms, Academic Press, Inc., 1985.
[5] Asada, G., Dong, M., Lin, T.S., Newberg, F., Pottie, Kaiser, W.J., and Marcy, H.O., Wireless Integrated Network Sensors: L ow Power Systems on a Chip, Proceedings of the 1998 European Solid State Circuits Conference.

[6] Bar-Yehuda, R., Goldreich, O., and Itai, A., Efficient emulation of single-hop radio network with collision detection on multi-hop radio network with no collision dete ction Distributed Computing, 5, (1991), 67-71.

[7] Bennett, F., Clarke, D., Evans, J. B., Hopper, A., Jones, A., Leask, D., Pic onet- Embedde dMobile Networking, IEEE Personal Communications, Vol 4 No 5, October 1997, pp 8-15.

[8] Bertzekas, D., and Gallager, R., Data Networks, Second Edition, Prentice-Hall, 1992.

[9] Betcher, K. E., Sorting Networks and Their Applications in Proc. AFIPS 1968 SJCC, vol. 32, Montvale, NJ:AFIPS Press, pp. 307-314.

[10] Bhuvaneswaran, R. S., Bordim, Jacir L., Cui, J., and Nakano, K.,F undamental Pr oto colsfor Wireless Sensor Networks, International P arallel and Distributed Processing Symposium (IPDPS), April 2001.

[11] Estrin, D., Govindan, R., Heidemann, J., and Kumar, S.,Next Century Challenges: Scalable Co ordination in Sensor Networks. In Proceedings of the Fifth Annual International Conference on Mobile Computing and Net works (MobiCOM '99), Seattle, Washington, August 1999.

[12] John Heidemann and Nirupama Bulusu, Using Geosp atial Information in Sensor Networks, In Proceedings of the Workshop on Intersections betw een Geospatial Information and Information T echnology Arlington, V A, USA, National Researc h Council. October, 2001.

[13] Kaplan, E. D., Understanding GPS: principles and applications, Artech House, Greenwich, 1998.

[14] Miller, R., and Stout, Q. F., Parallel Algorithms for $R$ egular Achite ctures: Meshes and Pyr amids The MIT Press, 1996.

[15] Nassimi, D. and Sahni, S., Bitonic Sort on MeshConnected Computer, IEEE Transactions on Computers, vol. c-27, NO. 1, January 1979.

[16] Thompson, C. D., and Kung, H. T., Sorting on a Mesh-Connected Parallel Computer, Communications of the ACM, vol 20, NO. 4, April 1977. 